\title{
Pellino3 targets the IRF7 pathway and facilitates autoregulation of TLR3- and viral-induced expression of type I interferons
}

\author{
Jakub Siednienko ${ }^{1,4}$, Ruaidhri Jackson ${ }^{1,4}$, Mark Mellett ${ }^{1,4}$, Nezira Delagic $^{1}$, Shuo Yang ${ }^{1}$, Bingwei Wang ${ }^{1}$, \\ Lisa S Tang1 ${ }^{1}$, John J Callanan ${ }^{2,3}$, Bernard P Mahon ${ }^{1} \&$ Paul N Moynagh $^{1}$
}

\begin{abstract}
Toll-like receptors (TLRs) sense pathogen-associated molecules and respond by inducing cytokines and type I interferon. Here we show that genetic ablation of the E3 ubiquitin ligase Pellino3 augmented the expression of type I interferon but not of proinflammatory cytokines in response to TLR3 activation. Pellino3-deficient mice had greater resistance against the pathogenic and lethal effects of encephalomyocarditis virus (EMCV). TLR3 signaling induced Pellino3, which in turn interacted with and ubiquitinated TRAF6. This modification suppressed the ability of TRAF6 to interact with and activate IRF7, resulting in downregulation of type I interferon expression. Our findings highlight a new physiological role for Pellino3 and define a new autoregulatory network for controlling type I interferon expression.
\end{abstract}

Recognition of pathogen-associated molecules in microbes by TLRs leads to activation of transcription factors such as NF- $\kappa \mathrm{B}$ that promote increased transcription of proinflammatory cytokines and interferons ${ }^{1}$. All mammalian TLRs, with the exception of TLR3, use the adaptor MyD88 as the receptor-proximal signaling molecule to trigger downstream activation of $\mathrm{NF}-\mathrm{\kappa B}^{2}$. The association of MyD88 with TLRs facilitates recruitment of members of the IRAK family of kinases that in turn activate the E3 ubiquitin ligase TRAF6 (refs. 3-5). The formation of polyubiquitin chains by TRAF6 serves to bring TAK1 into close proximity with its substrates, including I $\mathrm{KB}$ kinases (IKKs). The TAK1-induced phosphorylation and activation of IKK $\alpha$ and IKK $\beta$ promotes IKK-induced phosphorylation of I $\kappa$ B proteins ${ }^{6}$ that normally sequester NF- $\kappa B$ in an inactive form in the cytoplasm. Phosphorylated forms of $\mathrm{I} \kappa \mathrm{B}$ are subject to polyubiquitination and subsequently proteasome-dependent degradation, thus liberating $\mathrm{NF}-\kappa \mathrm{B}$ to translocate to the nucleus and transcriptionally upregulate the expression of a plethora of genes ${ }^{7}$. Most TLRs use this MyD88dependent pathway to activate NF- $\kappa B$, but TLR4 can additionally deploy another adaptor protein, TRIF, to trigger a MyD88-independent pathway that also activates NF- $\kappa B^{8}$. Among TLRs, TLR3 uses TRIF as its exclusive receptor-proximal adaptor protein. TRIF interacts with RIP1 kinase to trigger downstream IKK-mediated activation of NF- $\kappa B^{9,10}$. TRAF6 has been reported to associate with TRIF and mediate activation of NF- $\kappa B^{11-13}$, but other studies had concluded that TRAF6 is dispensable for TLR3 signaling ${ }^{14,15}$. Such discrepancies in relation to the role of TRAF6 in TRIF signaling may be due to cell-specific roles for TRAF6 and/or functional redundancy of TRAF6 with other members of the TRAF family ${ }^{11}$. In addition to activation of NF- $\kappa B$, TRIF can also trigger activation of interferon-regulatory factor (IRF) transcription factors. Thus, TRIF forms a complex with the kinases TBK1 and IKKi (also known as IKKe) and both kinases can catalyze phosphorylation and activation of IRF3 and IRF7, leading to their nuclear translocation and induction of type I interferons ${ }^{1,16}$. The latter are key antiviral molecules that block viral replication ${ }^{17,18}$.

It is clear from the above that ubiquitination is important in TLR signal transduction. Additionally, there is an emerging appreciation of the roles of the E3 ubiquitin ligase family of Pellino proteins in TLR signaling. The mammalian Pellino family consists of four members: Pellino1, Pellino2 and splice variants of Pellino3 termed Pellino3 long (Pellino3L; also known as Pellino3a) and Pellino3 short (Pellino3S; also known as Pellino3b) ${ }^{19,20}$. Each Pellino family member contains an $\mathrm{N}$-terminal forkhead-associated (FHA) domain that recognizes phosphothreonine residues and mediates association with IRAKs ${ }^{21}$, and a C-terminal RING-like domain that confers E3 ubiquitin ligase activity and an ability to catalyze lysine 63 (Lys63)-linked polyubiquitination of IRAKs ${ }^{22-24}$. Pellino proteins are subject to various forms of post-translational modification, including phosphorylation ${ }^{24-28}$, ubiquitination ${ }^{23-25}$ and sumoylation ${ }^{29}$ with phosphorylation being especially important for enhancing the E3 ligase activity. Pellino1 is regulated at the transcriptional level by TRIF signaling, which induces Pellino1 expression in an IRF3-dependent manner ${ }^{26}$. Although each of the Pellino proteins has most of the above features, there are unique functional differences. Pellino1 has been shown to act as a mediator in the NF- $\mathrm{KB}$ pathway ${ }^{30}$, whereas Pellino2 and Pellino3 have been associated with activation of MAPK pathways ${ }^{28,31-34}$. The generation of Pellino1-deficient mice had revealed a role for Pellino1 as the E3 

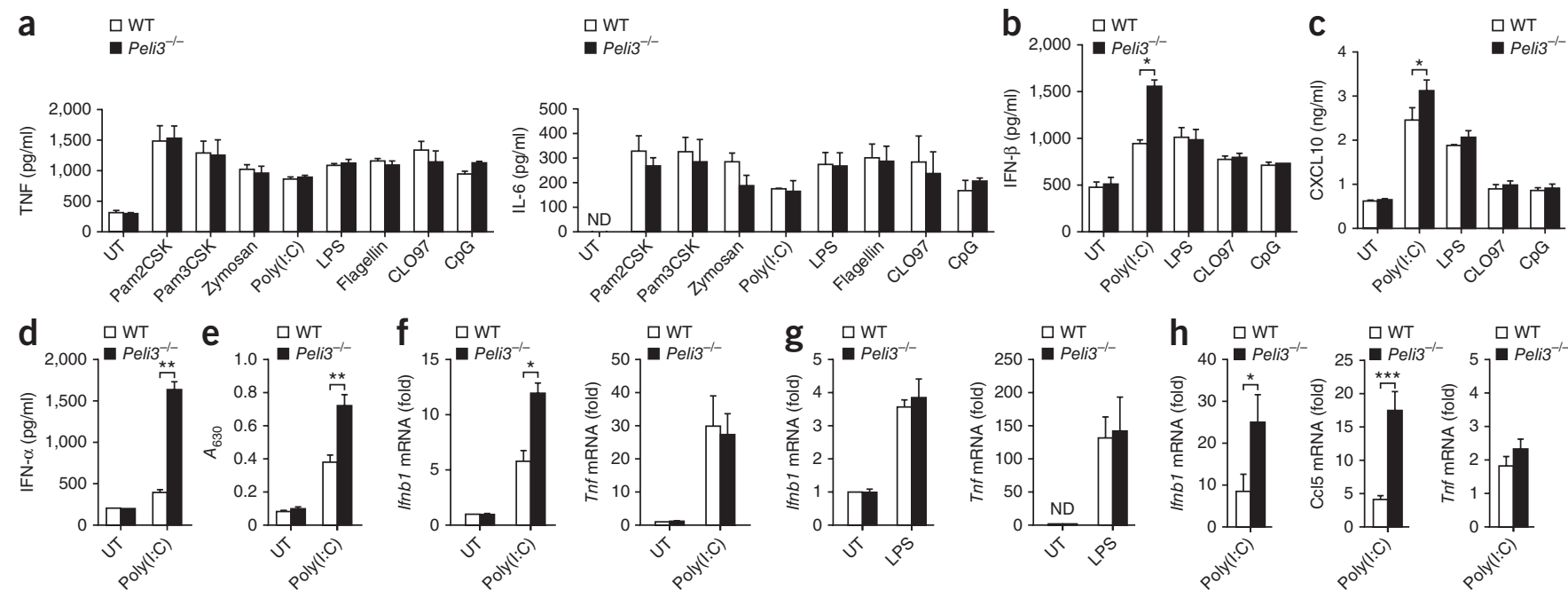

Figure 1 Pellino3 deficiency results in enhanced TLR3-induced expression of IFN- $\beta$ and related genes. (a) ELISA of TNF and IL-6 expression in medium from BMDMs isolated from wild-type (WT) and Pellino3-deficient (Peli3-l-) mice and treated with $10 \mathrm{ng} / \mathrm{ml}$ Pam2CSK, $10 \mathrm{ng} / \mathrm{ml} \mathrm{Pam3CSK}$, $1 \mu \mathrm{g} / \mathrm{ml}$ zymosan, $5 \mu \mathrm{g} / \mathrm{ml}$ poly(I:C), $10 \mathrm{ng} / \mathrm{ml}$ lipopolysaccharide (LPS), $1 \mu \mathrm{g} / \mathrm{ml}$ flagellin, $1 \mu \mathrm{g} / \mathrm{ml}$ CLO97 and $2 \mu \mathrm{g} / \mathrm{ml}$ CpG for $24 \mathrm{~h}$. UT, untreated.

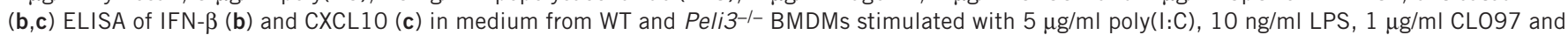

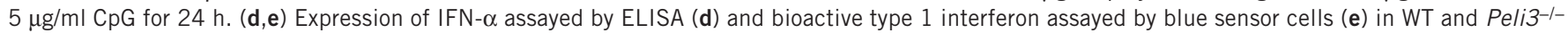
BMDMs stimulated with $5 \mu \mathrm{g} / \mathrm{ml}$ poly $(\mathrm{I}: \mathrm{C})$ for $24 \mathrm{~h}$. (f,g) Quantitative PCR of mRNA expression for Ifnb1 and Tnf in WT and Peli3-l- BMDMs that were

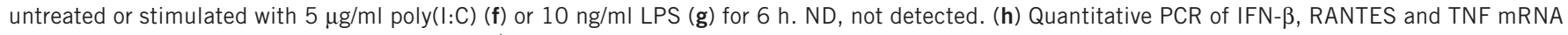
expression in PECs isolated from WT and Peli3 ${ }^{-/-}$mice $48 \mathrm{~h}$ after intraperitoneal injection with $1 \mathrm{mg} / \mathrm{kg}$ poly(I:C). Data are representative of three experiments (error bars, s.e.m.; $n=3$ ). ${ }^{*} P<0.05,{ }^{* *} P<0.01$ and ${ }^{* * *} P<0.001$ (paired Student's $t$-test).

ubiquitin ligase for RIP1 and a critical mediator of TRIF-dependent activation of NF- $\kappa \mathrm{B}$ in TLR3 and TLR4 pathways ${ }^{35}$. Pellino1 has been shown to negatively regulate $\mathrm{T}$ cell activation and suppress autoimmunity by promoting ubiquitination and proteolysis of the NF- $\mathrm{KB}$ subunit c-Rel in activated $\mathrm{T}$ cells $\mathrm{s}^{36,37}$. To date the physiological roles of Pellino2 and Pellino3 remain to be defined.

We report the generation of Pellino3-deficient mice and describe a new physiological role for Pellino3 in TLR signaling. We show that Pellino3 is dispensable for TLR-induced expression of proinflammatory cytokines but has a negative regulatory role in TLR3and viral-induced expression of type I interferon and related genes. Mechanistically, we show that TRIF signaling induces Pellino3 expression, which in turn inhibits the ability of TRAF6 to activate IRF7. These findings reveal Pellino3 as an important player in a new autoregulatory system for controlling type I interferon expression.

\section{RESULTS}

\section{Pellino3 regulates TLR3 induction of type I interferon}

We generated Pellino3-deficient mice with a floxed Peli3 allele (Supplementary Fig. 1a). We detected wild-type and recombined alleles by PCR analysis (Supplementary Fig. 1b). Semi-quantitative and real-time RT-PCR analysis of mRNA isolated from mouse embryonic fibroblasts (MEFs) demonstrated absence of Pellino3 mRNA expression in cells from Peli3 ${ }^{-/-}$mice (Supplementary Fig. 1c,d). Initially we used bone marrow-derived macrophages (BMDMs) from wild-type and Peli3 ${ }^{-/-}$mice to assess the effects of Pellino3 deficiency on TLR signaling. The ability of various TLR ligands to induce proinflammatory cytokines, such as TNF and IL-6, was comparable in BMDMs from wild-type and Pellino3-deficient mice as determined by enzyme-linked immunosorbent assay (ELISA; Fig. 1a), indicating a nonessential role for Pellino3 in mediating TLR-induced expression of proinflammatory cytokines. Various TLR ligands were similarly effective in inducing TNF in MEFs isolated from wild- type and Peli3 ${ }^{-/-}$mice (Supplementary Fig. 2a). Given that TLRs are highly expressed in various dendritic cell (DC) populations, we characterized the effects of Pellino3 deficiency on IL-6 expression in response to TLR3, TLR4 and TLR9 signaling in myeloid DCs (mDCs) and plasmacytoid DCs (pDCs; Supplementary Fig. 2b,c). Absence of Pellino3 had no effect on the TLR-mediated induction of IL-6 in these DC populations.

We next examined a potential role of Pellino3 in regulating the expression of type I interferons and interferon-stimulated genes, such as the chemokine CXCL10. We stimulated BMDMs with ligands for TLR3, TLR4, TLR7 and TLR9 because these are the predominant TLR family members that induce type I interferons, and measured the induction of interferon beta (IFN- $\beta$ ). Each of the ligands induced IFN- $\beta$ in wild-type BMDMs, but the TLR3 agonist polyriboinosinic: polyribocytidylic acid (poly(I:C)) was unique in that its efficacy in inducing IFN- $\beta$ was augmented in Pellino3-deficient BMDMs compared with wild-type BMDMs (Fig. 1b). Peli3 ${ }^{-1-}$ cells also exhibited selective enhancement of poly(I:C)-induced expression of CXCL10 (Fig. 1c). Poly(I:C) similarly induced higher IFN- $\alpha$ expression in Peli3 $^{-1-}$ BMDMs relative to their wild-type counterparts (Fig. 1d). The type I interferon produced in these cells was bioactive, as shown in a type I interferon reporter cell line (Fig. 1e). We also measured type I interferon and CXCL10 induction in MEFs, mDCs and pDCs stimulated with various TLR ligands (Supplementary Fig. 2). We observed enhanced induction of type I interferon and CXCL10 by poly(I:C) in Pellino3-deficient MEFs and mDCs. This effect was mediated by increased gene transcription because poly(I:C) induced greater amounts of Ifnb1 mRNA in Pellino3-deficient BMDMs relative to wild-type cells, whereas poly(I:C)-mediated induction of Tnf mRNA was independent of Pellino3 deficiency (Fig. 1f). The regulatory effects of Pellino3 on type I interferon expression seemed to be restricted to TLR3 because Pellino3 deficiency did not affect LPSmediated induction of $I f n b 1$ or Tnf mRNA in BMDMs (Fig. 1g). 
Figure 2 Pellino3-deficient cells exhibit an enhanced type 1 interferon response to EMCV infection. (a,b) ELISA of IFN- $\beta$, IFN- $\alpha$, CXCL10 and TNF expression in medium from BMDMs (a) or MEFs (b) isolated from wild-type (WT) and Peli3 ${ }^{-1-}$ mice and infected with EMCV (250 PFU)) for the indicated durations. (c,d) Expression of bioactive type 1 interferon assayed in blue sensor cells (c) and quantitative PCR of Ifnb1, Cxcl10, Ifna and Tnf mRNA expression (d) in WT and Peli3-/MEFs infected with EMCV (250 PFU) for the indicated durations. Error bars, s.e.m. ( $n=3$ experiments). NS, not significant, ${ }^{*} P<0.05$, ${ }^{* *} P<0.01$ and ${ }^{* * *} P<0.001$ (paired Student's $t$-test).

To confirm the regulatory effects of Pellino3 in poly(I:C) signaling in vivo, we injected wild-type and Pellino3-deficient mice intraperitoneally with poly(I:C), isolated peritoneal exudate cells (PECs) and assayed them for expression of genes encoding IFN- $\beta$, RANTES and TNF (Ifnb1, Ccl5 and Tnf, respectively). The chemokine RANTES was included in this analysis because, similar to type I interferon, its expression is regulated by IRFs. In vivo administration of poly(I:C) induced expression of Ifnbl and Ccl5 in wild-type mice, and this was enhanced in Pellino3-deficient mice (Fig. 1h). In contrast, poly(I:C)-mediated induction of $\operatorname{Tnf}$ was similar in wild-type and Peli3 ${ }^{-/-}$mice, indicating that Pellino3 selectively regulates expression of type I interferons and related genes without affecting expression of proinflammatory genes.

Given that poly(I:C) can stimulate both TLR3 and the cytosolic receptor MDA-5 (ref. 38), we investigated which of the two pathways is potentially modulated by Pellino3 in this experimental system. We observed loss of induction of Ifnb1, Ccl5 and Tnf mRNAs, which encode IFN- $\beta$, RANTES and TNF, respectively, in response to poly (I:C) in TRIF-deficient BMDMs but not in BMDMs deficient in the signaling adaptor MAVS (Supplementary Fig. 3a). Given the role of TRIF in TLR3 signaling and MAVS in MDA-5 signaling, these results provide evidence that TLR3 is the primary sensing receptor for poly(I:C) in BMDMs. In addition, we applied poly(I:C) to cells directly, which favors stimulation of TLR3, whereas MDA-5-mediated responses to poly(I:C) require transfection of cells for delivery of the synthetic dsRNA to the cytosol. To confirm that Pellino3 targets TLR3 in our model, we tested the effect of direct application or liposome-mediated delivery of poly(I:C) to wild-type and Pellino3-deficient BMDMs on IFN- $\beta$ expression. Both delivery methods induced IFN- $\beta$ in wild-type BMDMs. IFN- $\beta$ induction was enhanced in Pellino3-deficient cells in response to direct application of poly(I:C) but not in response to transfection-mediated delivery (Supplementary Fig. 3b). These findings suggest that Pellino3 selectively targets the TLR3 pathway.

We next addressed the regulatory effects of Pellino3 on TLR3induced expression of type I interferon and related genes in human cells. We established U373 cell lines transduced with lentivirus to stably express PELI3-specific short hairpin RNA (shRNA) or a control shRNA. We confirmed knockdown of Pellino3 expression by RTPCR (Supplementary Fig. 4a). Expression of IFNB1, IFNA1, CCL5 and CXCL10 mRNA, in response to poly(I:C) stimulation for various times, was greatly enhanced under conditions of Pellino3 knockdown (Supplementary Fig. 4b-e). We also observed enhanced expression at the protein level (Supplementary Fig. 4f,g). Poly(I:C)-induced expression of IL6 mRNA or protein were not affected in Pellino3 knockdown U373 cells (Supplementary Fig. 4h,i). Together, these results indicate a selective role for Pellino3 as a negative regulator of TLR3-induced expression of type I interferon but not of proinflammatory genes.

\section{Enhanced protection from EMCV infection in Peli3-/- mice}

Given that poly(I:C) is a synthetic analog of viral dsRNA, we investigated the role of Pellino3 in regulating the host response to EMCV,

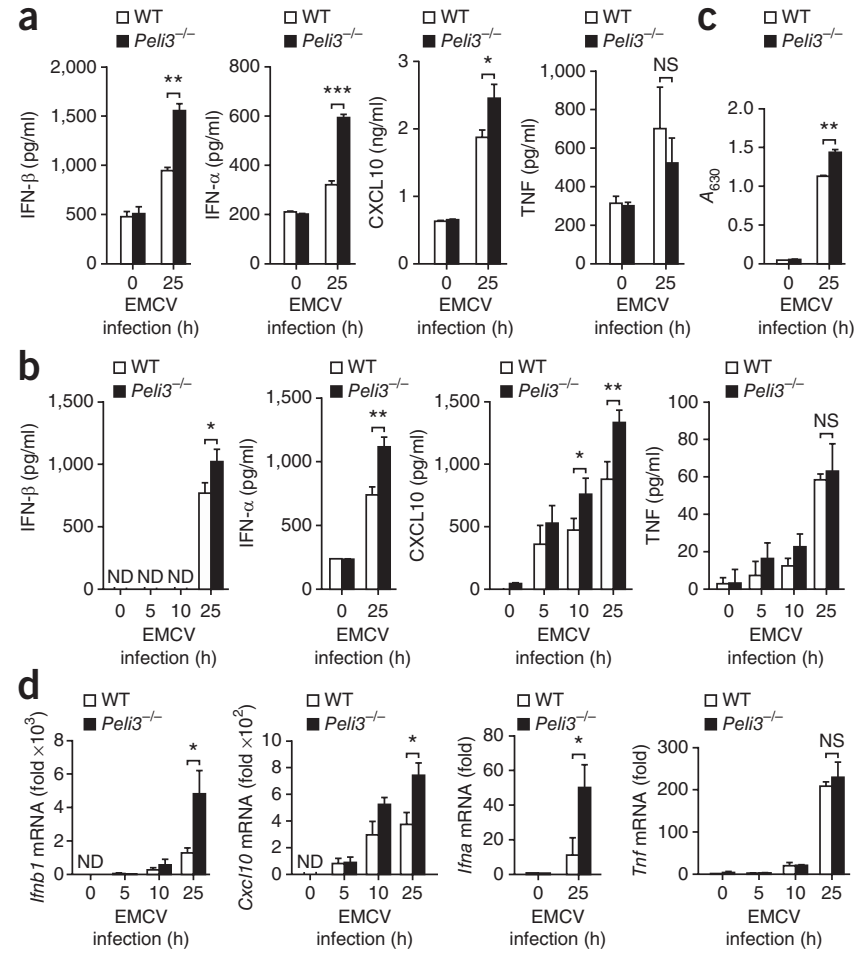

which generates dsRNA as part of its life cycle and for which TLR3 signaling has been shown to be important for a protective response $\mathrm{e}^{39}$. EMCV induced IFN- $\beta$, IFN- $\alpha$, CXCL10 and TNF in wild-type BMDMs. Whereas expression of type I interferon and CXCL10 was additionally enhanced in Peli3 ${ }^{-1-}$ cells, EMCV-induced TNF was not affected by Pellino3 deficiency (Fig. 2a). We obtained similar results in Pellino3-deficient MEFs (Fig. 2b). The enhanced production of type I interferons was bioactive (Fig. 2c). Pellino3-deficient MEFs showed enhanced induction of the mRNAs encoding IFN- $\beta$, IFN- $\alpha$ and CXCL10 in response to EMCV challenge relative to wildtype cells (Fig. 2d). Loss of Pellino3 had no effect on the Tnf mRNA induced by EMCV, suggesting Pellino3 specifically regulates type I interferon gene expression in response to EMCV.

We next investigated the physiological and pathological relevance of these regulatory effects of Pellino3 in the context of EMCV infection with two lethal viral doses in wild-type and $\mathrm{Peli3}^{-/-}$mice. The higher infection dose of 250 plaque-forming units (PFU) per mouse had a lethal effect in all wild-type mice $7 \mathrm{~d}$ after infection (Fig. 3a), whereas $60 \%$ of Pellino3-deficient mice survived and remained healthy for the duration of the infection study. A lower dose of EMCV (50 PFU per mouse) caused 50\% lethality in wildtype mice $10 \mathrm{~d}$ after infection, whereas all Pellino3-deficient mice survived (Fig. 3a). An increased heart/body weight ratio, characteristic of EMCV infections, was clearly evident in wild-type mice but not in Pellino3-deficient mice at low doses of infection (Fig. 3b). Examination of heart tissue from EMCV-infected wild-type mice by microscopy revealed histopathological changes with random multifocal mononuclear interstitial infiltrates as well as myofiber nuclear karyolysis and myofiber cell loss accompanied by mild edema (Fig. 3c). We did not observe these changes in the heart tissue from EMCV-infected Peli3 ${ }^{-/-}$mice. Enhanced protection from the pathological and lethal effects of EMCV infection in Pellino3deficient mice was associated with a lower viral load in the hearts, as measured by expression of the replicase gene from EMCV (Fig. 3d). 

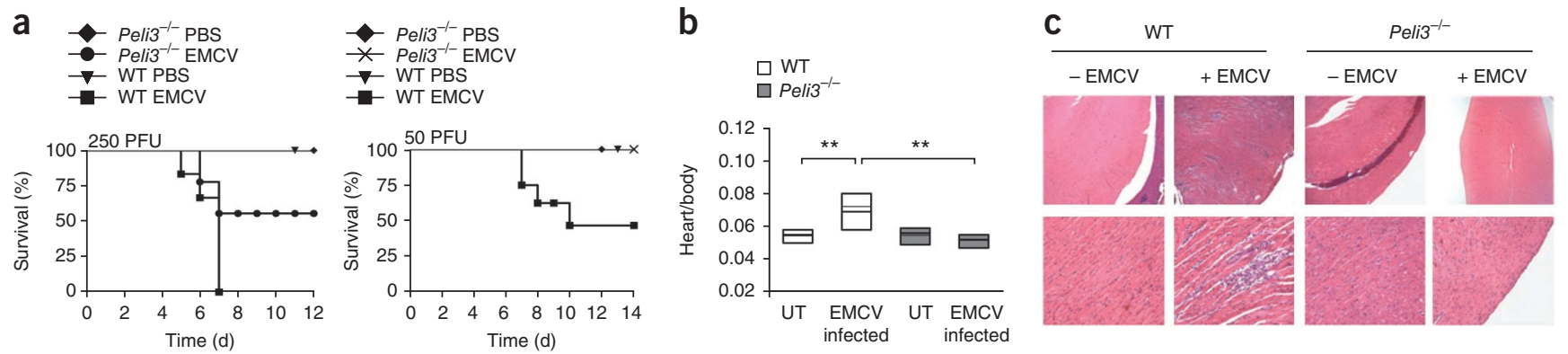

Figure 3 Peli3-/- mice exhibit augmented IFN- $\beta$ expression and increased viral clearance in response to EMCV infection. (a) Survival rates of age-matched and sex-matched wild-type (WT; $n=7)$ and Peli3-l- $(n=7)$ mice after intraperitoneal injection with 250 PFU or 50 PFU of EMCV virus or with PBS as a vehicle control ( $P=0.005$, Kaplan-Meier analysis). Moribund mice were killed. Surviving mice were killed $12 \mathrm{~d}$ or $14 \mathrm{~d}$ after infection. (b,c) Heart/body weight ratios (b) and heart samples stained with hematoxylin and eosin (c) from WT and Peli3-1- mice after intraperitoneal injection with EMCV (50 PFU). UT, untreated. Original magnification, $\times 40$ (top) and $\times 200$ (bottom). (d-f) Quantitative PCR of mRNA expression for EMCV replicase (d), Ifnbl (e) and Tnf (f) in heart tissue from WT and Peli3-/- mice $4 \mathrm{~d}$ after intraperitoneal injection with 50 PFU EMCV ( $n=3$ mice each group). Error bars, s.e.m. ( $n=3$ experiments). ${ }^{*} P<0.01$ (paired Student's $t$-test).
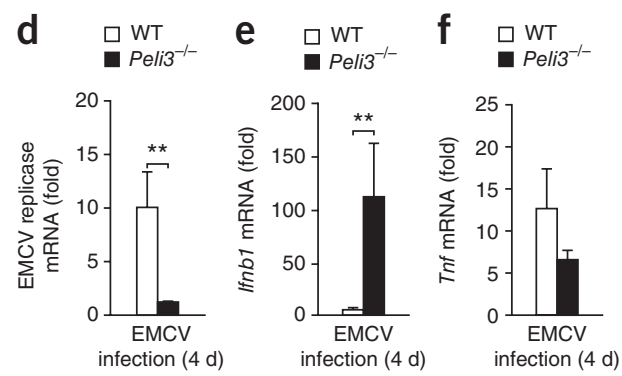

This was consistent with enhanced IFN- $\beta$ expression in the hearts from EMCV-infected Peli3 ${ }^{-/}$mice (Fig. 3e), which presumably facilitated more efficient clearance of the virus. Expression of TNF in the heart of EMCV-infected Peli3 ${ }^{-/}$mice was lower than that in infected wild-type mice (Fig. 3f), correlating with the lower grade of inflammation suggested by the histochemical analysis (Fig. 3c). Thus, Pellino3 has an important regulatory function in controlling type I interferon expression in response to viral challenge.

\section{Pellino3 selectively regulates activation of IRF7}

Because the type I interferon and related genes that are inhibited by Pellino 3 are strongly regulated by NF- $\kappa \mathrm{B}$ and IRFs, we compared these pathways in response to poly(I:C) in wild-type and Pellino3deficient cells. The absence of Pellino3 did not affect the capacity of poly(I:C) to induce phosphorylation of IKKs or of their substrate $\mathrm{I} \kappa \mathrm{B} \alpha$, both of which are activation indices of the canonical NF- $\kappa \mathrm{B}$ pathway (Fig. 4a). To investigate the activation of IRFs, we assessed the binding of nuclear extracts to an oligonucleotide containing the IRF recognition motif from the positive regulatory domains I/III (PRD I/III) of the enhancer region of the Ifnbl gene. Poly(I:C) induced a modest and time-dependent increase in the binding of nuclear extract proteins from wild-type cells to the PRD I/III motif, whereas binding was enhanced in extracts from Pellino3-deficient cells (Fig. 4b), suggestive of a role for Pellino3 in targeting the IRF arm of the TLR3 pathway. However, loss of Pellino3 did not affect the ability of poly(I:C) to promote phosphorylation of IRF3 or its upstream kinase TBK1 (Fig. 4c). Given that IRF7 can also bind to the PRD I/III domain, we probed the regulatory effect of Pellino3 on the phosphorylation of IRF7, which is required for its activation. Because commercially available phosphospecific IRF7 antibodies are only sufficiently sensitive to detect overexpressed IRF7, we characterized the poly(I:C)-induced phosphorylation of IRF7 expressed in HEK293 cells, stably expressing TLR3 and transfected with shRNA targeting PELI3. In this system, poly(I:C) promoted time-dependent phosphorylation of IRF7 in cells transfected with a control shRNA, and this was enhanced in Pellino3-knockdown cells (Fig. 4d). We next probed the regulatory effect of Pellino3 on the phosphorylation of endogenous IRF7. Given the lack of sensitivity of the phospho-IRF7 antibody, we immunoblotted for serine phosphorylation of IRF7 immunoprecipitated from mDCs that had been stimulated with poly(I:C). The amount of serine-phosphorylated IRF7 was higher in Pellino3-deficient mDCs relative to that in wild-type mDCs (Fig. 4e).

Because phosphorylation of IRF7 is a prerequisite for its nuclear translocation, we next assessed the effects of Pellino3 deficiency on the nuclear localization of IRF7. We isolated nuclear fractions from wild-type and Pellino3-deficient BMDMs that had been stimulated with poly(I:C) for various durations. Time-dependent poly(I:C)induced nuclear localization of IRF7 was stronger in Peli3 ${ }^{-1-}$ than in wild-type BMDMs (Fig. 4f). Whereas poly(I:C) also caused increased nuclear localization of IRF3, this was not affected by the absence of Pellino3. We used confocal microscopy to complement these observations on the subcellular localization of IRF7. In unstimulated MEFs from wild-type mice, IRF7 localized to the cytoplasm, and stimulation with poly(I:C) promoted some nuclear accumulation of IRF7 (Fig. 4g). However, the effect of poly(I:C) in Pellino3-deficient MEFs was more notable, with all detectable IRF7 translocating to the nucleus after poly(I:C) stimulation. Finally, we assessed whether the enhanced poly(I:C)-induced nuclear localization of IRF7 in Pellino3-deficient cells translated into increased binding of IRF7 to responsive promoters. We stimulated wildtype and Pellino3-deficient BMDMs with poly(I:C) and assayed the in vivo binding of IRF7 to the Ifnb1 promoter by chromatin immunoprecipitation. Poly(I:C)-induced binding of IRF7 to the Ifnb promoter was increased in Pellino3-deficient cells compared to that in wild-type BMDMs (Fig. 4h).

All of the above data strongly indicate that Pellino3 negatively regulates activation of IRF7 but not of IRF3. We directly addressed this by assessing the effects of overexpressed Pellino3 on the induction of a cotransfected IRF3-regulated or IRF7-regulated reporter gene. Using this system, both spliced forms of Pellino3 inhibited poly(I:C)induced activation of IRF7 but not of IRF3 (Supplementary Fig. 5a,b), consistent with Pellino3 targeting the IRF7 pathway. We also characterized the regulatory effects of the spliced forms of Pellino3 on naturally occurring IRF7-responsive promoters. Both spliced forms inhibited poly(I:C)-induced activation of the 

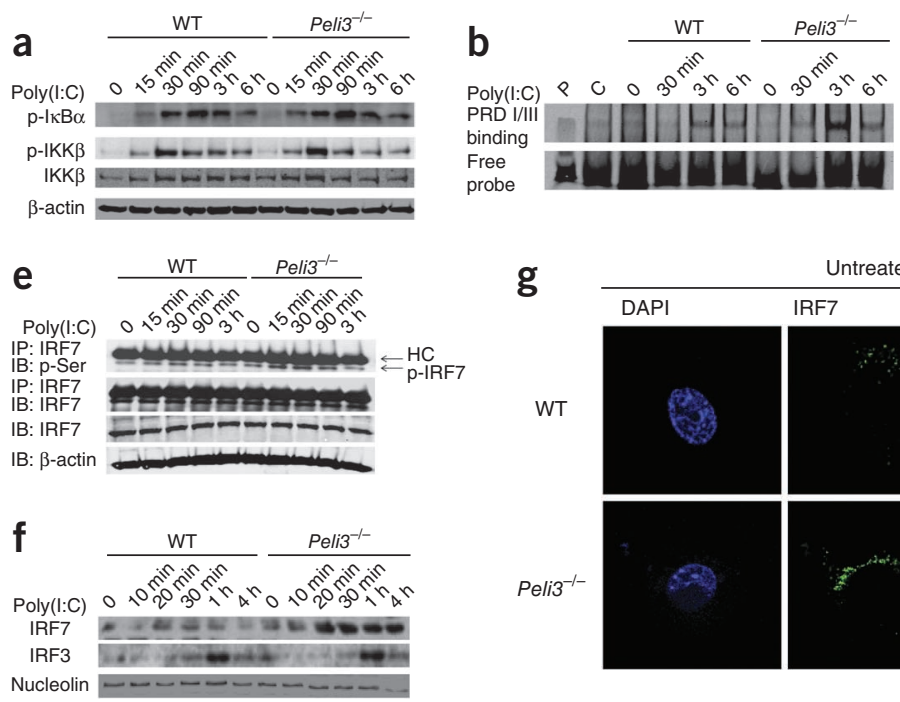

g

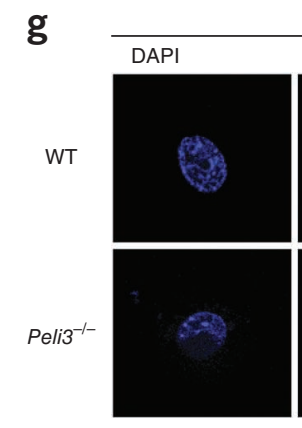

Untreated
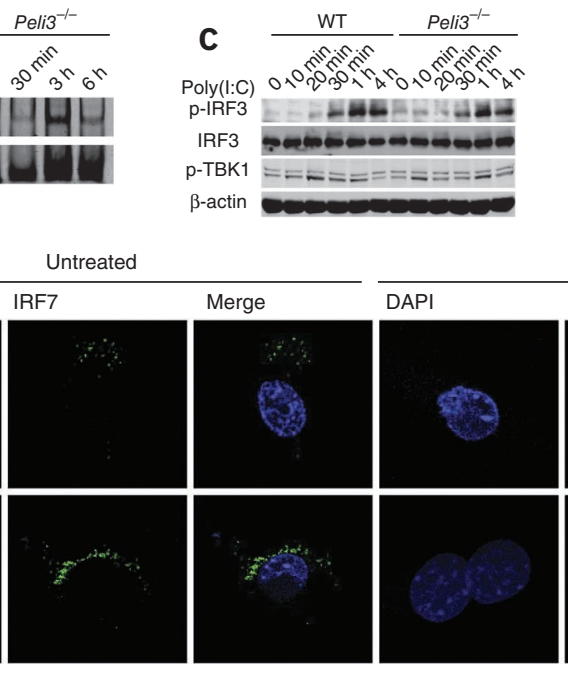

d

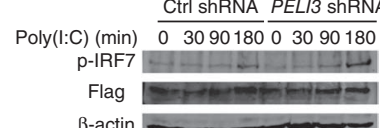

$\beta$-actin

Poly(l:C) (90 min)
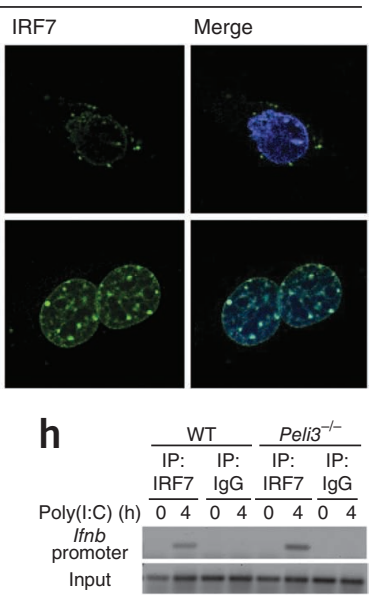

Figure 4 Pellino3 inhibits activation and nuclear translocation of IRF7. (a) Immunoblot analysis of phosphorylated ( $\mathrm{p}-) \mid \mathrm{KB}-\alpha, \mathrm{p}-\mathrm{IKK} \beta$ and total IKK $\beta$ in lysates of wild-type (WT) and Peli3-1- BMDMs stimulated with $5 \mu \mathrm{g} / \mathrm{ml}$ poly $(\mathrm{I}: \mathrm{C})$ for indicated durations. $\beta$-actin was used as a loading control. (b) Electrophoretic mobility shift assay analysis of binding of an oligonucleotide, containing the consensus PRD I/III domain of the Ifnbl promoter, to nuclear extract proteins from WT and Peli3-/- MEFs stimulated with $25 \mu \mathrm{g} / \mathrm{ml}$ poly(I:C) for indicated durations. Controls: oligonucleotide probe run by itself (P) and nuclear extracts (from Peli3-/- cells stimulated with poly (I:C) for $3 \mathrm{~h}$ ) preincubated for 15 min with the unlabeled competing oligonucleotide before assaying for binding to the labeled PRD I/III oligonucleotide (C). (c) Immunoblot analysis as in a of p-IRF3, p-TBK1 and total IRF3. (d) Immunoblot analysis of p-IRF7 and Flag-tagged IRF7 in lysates of $25 \mu \mathrm{g} / \mathrm{ml}$ poly(I:C)-treated HEK293 cells stably expressing TLR3 and previously transfected with an IRF7-Flag expression construct and control or PEL/3-specific shRNA. (e) Immunoblot (IB) analysis of phosphoserine (p-Ser) and IRF7 in Iysates and immunoprecipitated (IP) IRF7 samples from WT and Peli3-l- mDCs stimulated with $5 \mu \mathrm{g} / \mathrm{ml}$ poly (I:C) for indicated durations. Arrows, IgG heavy chain (HC) and p-IRF7. (f) Immunoblot analysis of IRF7, IRF3 and nucleolin in nuclear extracts from WT and Peli3-l- BMDMs stimulated with $5 \mu$ g/mI poly(I: C) for indicated durations. (g) Confocal images obtained with a $\times 63$ oil-immersion objective (total magnification of images, $\times 630$ ) of WT and Peli3-l- MEFs stimulated with $25 \mu \mathrm{g} / \mathrm{ml}$ poly(I:C) for $90 \mathrm{~min}$. Cells were incubated with nuclei-staining DAPI. (h) Chromatin immunoprecipitation analysis of binding of IRF7 to the Ifnbl promoter in WT and Peli3 ${ }^{-/-}$BMDMs stimulated with $5 \mu \mathrm{g} / \mathrm{ml}$ poly(I:C) for $4 \mathrm{~h}$. Input DNA (before IRF7 immunopreciptiation) and immunoprecipitated (with anti-IRF7 (IP: IRF7) or mouse IgG (IP: IgG) chromatin were analyzed by PCR (25 cycles) with primers specific for the $I f n b 1$ promoter. Data are representative of three experiments.

IFNA4 and IFNB promoters (Supplementary Fig. 5c,d). Furthermore, given that IRF7 binds to the PRDI/III domains of the IFNB promoter, we confirmed that Pellino3 targets this region of the IFNB promoter (Supplementary Fig. 5e). These data suggest that Pellino3 regulates the nuclear translocation and activation of IRF7.

\section{Pellino3 regulates TRAF6-dependent ubiquitination of IRF7}

We next probed the mechanism by which Pellino3 can target the IRF7 pathway. Because loss of Pellino3 did not affect poly(I:C)induced phosphorylation of TBK1, a known activator of IRF7, we investigated the effects of Pellino3 deficiency on the ubiquitination status of IRF7. Previous studies have demonstrated that ubiquitination of IRF7 is a prerequisite for IKKi-mediated phosphorylation and activation of IRF7 (refs. 40,41). Poly(I:C) promoted a modest increase in the ubiquitination of IRF7 in MEFs from wild-type mice. This was considerably enhanced in MEFs from $\mathrm{Peli3}^{-/-}$mice (Fig. 5a). Given that TRAF6 has been shown to interact with and ubiquitinate IRF7 (refs. 40,41), we next probed the effects of Pellino3 on the TRAF6-IRF7 interaction. Poly(I:C) promoted the interaction of TRAF6 with IRF7 in wild-type MEFs, and this was augmented in Peli3 $^{-/}$MEFs, as shown by increased coimmunoprecipitation of TRAF6 and IRF7 (Fig. 5b). Poly(I:C) did not induce IFN- $\beta$ in MEFs from TRAF6-deficient mice (Fig. 5c), demonstrating a functional role for TRAF6 in poly(I:C)-induced expression of type I interferon. This deficiency in TLR3 signaling was rescued by reconstitution of
Traf6 ${ }^{-/-}$MEFs with wild-type TRAF6 but not with a point mutant form of TRAF6 (C70A) that obliterates its E3 ubiquitin ligase activity (Fig. 5d). These results are consistent with a model in which Pellino3 targets TRAF6-induced ubiquitination of IRF7 and IFN- $\beta$ expression downstream of TLR3.

\section{Pellino3 mediates ubiquitination of TRAF6}

We next investigated the regulatory process by which TLR3 deploys Pellino3 to negatively regulate TRAF6-mediated activation of IRF7. Poly(I:C) induced expression of Peli3 mRNA in wild-type BMDMs (Fig. 6a) but not in BMDMs lacking TRIF, TBK1 or IKKi. In addition, poly(I:C) promoted the interaction of Pellino3 with TRAF6 in a time-dependent manner, as evidenced by coimmunoprecipitation of the two proteins (Fig. 6b). We probed the functional consequence of this interaction by measuring the ubiquitination of TRAF6 in wild-type and Pellino3-deficient cells. Because Pellino3 is an E3 ubiquitin ligase, we especially investigated the ubiquitination of TRAF6. Stimulation with poly(I:C) caused ubiquitination of TRAF6 in wild-type MEFs but not in MEFs from Peli3-/- mice (Fig. 6c), indicating an important role for Pellino3 in the ubiquitination of TRAF6 downstream of TLR3. This was also the case in human cells, as HEK293 cells stably expressing TLR3 and transfected with PELI3specific shRNA did not exhibit increased ubiquitination of TRAF6 in response to poly(I:C), whereas cells transfected with control shRNAs strongly responded to poly(I:C) (Supplementary Fig. 6a). 
Figure 5 Loss of Pellino3 enhances binding of TRAF6 to IRF7 and ubiquitination of IRF7. (a,b) Immunoblot (IB) analysis of IRF7 and ubiquitin (a) or TRAF6 (b) in lysates and immunoprecipitated (IP) IRF7 samples from wild-type (WT) and Peli3-1- MEFs stimulated with $25 \mu \mathrm{g} / \mathrm{ml}$ poly (I:C) for indicated durations. $\beta$-actin was used as a loading control. (c) Quantitative PCR of mRNA expression for IFN- $\beta$ in WT, Peli3-/- and Traf6 $^{-/-}$MEFs stimulated or untreated (UT) with $25 \mu \mathrm{g} / \mathrm{ml}$ poly(I:C) for 6 h. (d) Quantitative PCR of mRNA expression for IFN- $\beta$ in Traf6 ${ }^{-1}$ MEFs transfected with an empty vector (EV) construct or expression plasmids encoding WT TRAF6 or TRAF6 (C70A) $(3 \mu \mathrm{g})$ and stimulated with $25 \mu \mathrm{g} / \mathrm{ml}$ poly (I:C) for $6 \mathrm{~h}$ or untreated (UT). Expression of TRAF6 constructs in Traf6-/- MEFs was determined by immunoblotting. Immunoprecipitation experiments $(\mathbf{a}, \mathbf{b})$ are representative of three experiments. Error bars (c,d), s.e.m. ( $n=3$ experiments). ${ }^{*} P<0.05,{ }^{* * *} P<0.001$ and NS, not significant (paired Student's t-test).

The responsiveness of $\mathrm{Peli3}^{-/-}$MEFs to poly(I:C)-induced ubiquitination of TRAF6 was restored by retrovirally mediated expression of mouse Pellino3 (Supplementary Fig. 6b). The E3 ligase activity of Pellino3 was directly required for the ubiquitination of TRAF6 because a mutant form of Pellino3 that lacks a functional RING domain did not reconstitute the poly(I:C)-induced ubiquitination of TRAF6 in Peli3 ${ }^{-1-}$ MEFs (Fig. 6d).

We next evaluated the functional link between the ability of Pellino3 to mediate poly(I:C)-induced ubiquitination of TRAF6 and the regulatory effects of Pellino 3 on IFN- $\beta$ expression. Reintroduction of mouse Pellino3 into Peli3 ${ }^{-/-}$MEFs blunted the ability of poly(I:C) to induce Ifnbl mRNA and IFN- $\beta$ protein, whereas the mutated form of Pellino3 with a nonfunctional RING domain did not exert this inhibitory influence (Fig. 6e). This also applies to human cells because exogenous expression of Pellino3 in HEK293 TLR3 cells expressing PELI3-specific shRNA inhibited poly(I:C)-induced expression of IFN- $\beta$, whereas the RING-mutated form of Pellino3 was ineffective (Supplementary Fig. 6c). We next

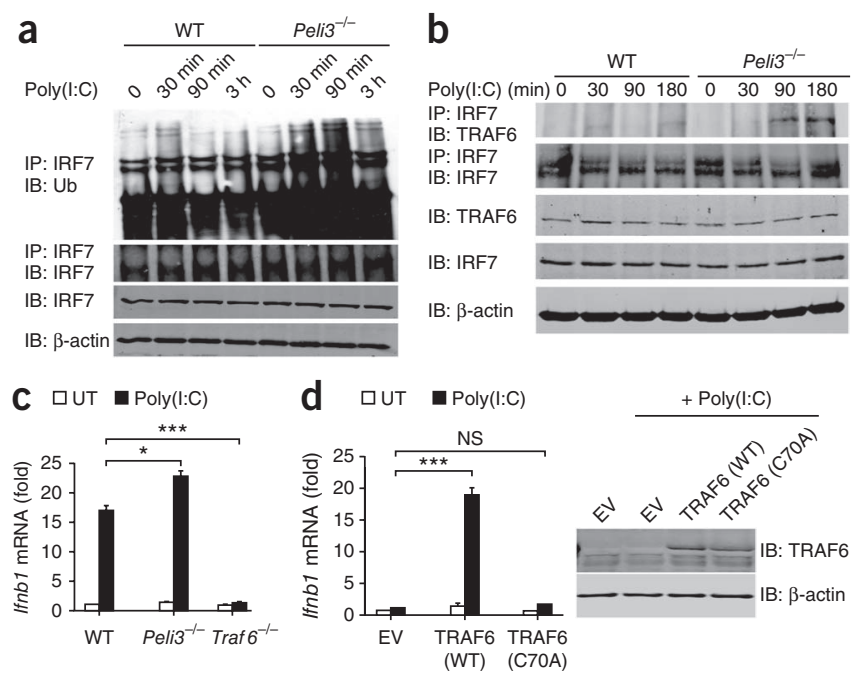

explored whether ubiquitination of TRAF6 affects its capacity to ubiquitinate IRF7. As expected, the coexpression of TRAF6 and IRF7 led to strong ubiquitination of IRF7, and this was lost with a point mutation $(\mathrm{C} 70 \mathrm{~A})$ of TRAF6 that interferes with the E3 ligase activity of TRAF6 (Fig. 6f). However, mutation of the primary ubiquitination site in TRAF6 (Lys124) led to slightly higher levels of ubiquitinated IRF7, consistent with the idea that ubiquitination of TRAF6 has an inhibitory effect on its E3 ubiquitin ligase activity. Furthermore, the TRAF6 mutant lacking the ubiquitination site was more effective than its wild-type counterpart in reconstituting poly(I:C)-induced expression of IFN- $\beta$ in TRAF6-knockdown cells (Fig. 6g). These data are consistent with a model where poly(I:C) induces Pellino3 expression and promotes its interaction with and a

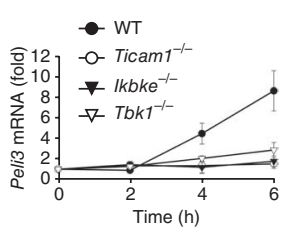

b

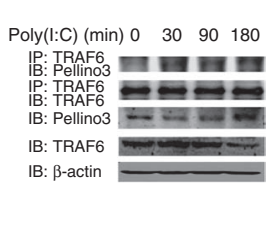

C

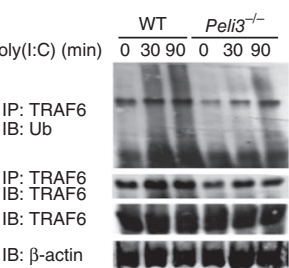

d

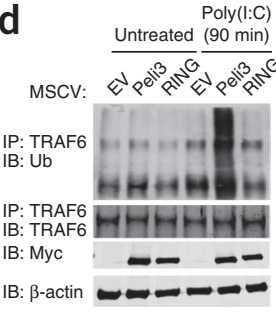

Figure 6 Pellino3 mediates TLR3-induced ubiquitination of TRAF6, which negatively regulates ubiquitination of IRF7 and expression of IFN- $\beta$. (a) Quantitative PCR analysis of Peli3 mRNA expression in immortalized BMDMs generated from wild-type (WT), Ticam $1^{-/-}, I \mathrm{kbke^{-/- }}$ and Tbk $1^{-1-}$ mice and stimulated with $5 \mu \mathrm{g} / \mathrm{ml}$ poly (I:C) for indicated durations. (b) Immunoblot (IB) analysis of Pellino3 and TRAF6 in lysates and immunoprecipitated (IP) TRAF6 samples from U373 cells (b) and of ubiquitin (Ub) and TRAF6 in lysates and immunoprecipitated (IP) TRAF6 samples from WT and Peli3-l- MEFs (c) stimulated with $25 \mu \mathrm{g} / \mathrm{ml}$ poly(I:C) for indicated durations. $\beta$-actin was used as a loading control. (d,e) IB analysis of ubiquitin and TRAF6 in immunoprecipitated (IP) TRAF6 samples (d), expression of Ifnb1 mRNA assayed by quantitative PCR (e, top) and bioactive type 1 interferon assayed by blue sensor cells (e, bottom) in untreated or $25 \mu \mathrm{g} / \mathrm{ml}$ poly(l:C)-treated WT and Peli3 ${ }^{-1-}$ MEFs, previously infected with MSCV retrovirus containing an empty vector (EV) plasmid or one encoding WT mouse (m)Pellino3 or mPellino3 with a mutated RING domain. (f) IB analysis of ubiquitin and IRF7 in immunoprecipitated (IP) IRF7 samples from HEK293 cells stably expressing TLR3 and previously transfected with constructs encoding Flag-tagged IRF7, WT TRAF6 TRAF6 (C70A) and TRAF6 (K124R) (1 $\mu \mathrm{g})$. (g) Quantitative PCR analysis of IFNB1 mRNA in untreated or $25 \mu \mathrm{g} / \mathrm{ml}$ poly(I:C)-treated HEK293 cells stably expressing TLR3 and previously transfected with TRAF6-specific or GAPDH-specific siRNA and expression plasmids encoding WT TRAF6, TRAF6(K124R) or an empty vector control (EV) control (1 $\mu \mathrm{g})$. Data are representative of three experiments $(\mathbf{b}-\mathbf{d}, \mathbf{f})$ or are presented as the mean \pm s.e.m. of at least three experiments $(\mathbf{a}, \mathbf{e}, \mathbf{g})$. NS, not significant, ${ }^{*} P<0.05$, $* * P<0.01$ and ${ }^{* * *} P<0.001$ (paired Student's $t$-test for $\mathbf{e}$ and ANOVA for $\mathbf{g}$ ). 
ubiquitination of TRAF6, thus negatively influencing the ability of the latter to ubiquitinate IRF7 and induce IFN- $\beta$ expression.

\section{DISCUSSION}

Using Pellino3-deficient mice, in this study we characterized the physiological role of Pellino3 in innate immunity. Peli3 ${ }^{-1-}$ mice were viable and developed normally. Our data indicate that Pellino3 does not act as a mediator of proinflammatory cytokine expression in response to TLRs but has a key regulatory role in controlling TRIFdependent type I interferon expression in the TLR3 pathway. We propose that TLR3 specifically uses Pellino3 as part of an autoregulatory signaling network in which stimulation of TLR3 by viruses or defined ligands, such as dsRNA, leads to upregulation of Pellino3, facilitating its interaction with TRAF6 and ubiquitination of the latter. We propose that this modification of TRAF6 serves to terminate its ability to ubiquitinate IRF7, thus shutting down a key driver of type I interferon expression.

In addition to delineating a new regulatory network that controls type I interferon expression, the findings also highlight a new function for Pellino proteins. Whereas overexpression and knockdown approaches have previously implicated Pellino proteins as regulators of the NF- $\kappa \mathrm{B}$ and MAP kinase pathways, studies in a physiological setting have been restricted to Pellino1 (refs. 35, 36). Using Pellino1-deficient mice, Pellino1 has been shown to regulate the NF- $\kappa \mathrm{B}$ pathway and act as a key mediator of TRIF-induced expression of proinflammatory cytokines in the TLR3 and TLR4 pathways. Our present studies show that Pellino3 regulates TRIF-dependent signaling in a manner that is very different from that of Pellino1, in that Pellino3 regulates TRIF-induced expression of IFN- $\beta$. This emphasizes divergent functions for different members of the Pellino family and highlights specific roles for Pellino proteins in these pathways.

We used EMCV as a naturally occurring agent that triggers antiviral immunity via TLR3. TLR3 signaling is essential for mediating cytokine expression, viral clearance and reducing lethality in response to EMCV infection ${ }^{39}$. Our findings indicate that absence of Pellino3 leads to enhanced viral clearance, diminished pathology and greater survival in mice, and this is consistent with greatly augmented TLR3induced expression of type I interferons in response to EMCV infection when Pellino3 is absent. This reaffirms a critical role for Pellino3 in tempering type I interferon expression in response to TLR3 stimulation. However, our findings cannot exclude a role for Pellino3 in similarly regulating the signaling of the viral sensing retinoic acidinducible gene (RIG-I)-like receptors, as Mda-5 has also been reported to detect poly(I:C) and act as a sensor of EMCV infection and as a mediator of EMCV-driven type I interferon expression ${ }^{38}$. However, responses to poly(I:C) mediated by $\mathrm{Mda}-5$ require cell transfection to facilitate delivery of the synthetic dsRNA to the cytosol-resident Mda-5. Under conditions of transfection-mediated delivery, we showed that loss of Pellino3 was without effect, strongly suggesting that Pellino3 targets the TLR3 pathway and not the Mda-5 pathway. Furthermore, using our delivery protocol, we observed loss of cytokine induction in response to poly(I:C) in TRIF-deficient BMDMs but not in MAVS-deficient BMDMs, reaffirming that Pellino3 is targeting the TLR3 pathway.

In our model, Pellino3 negatively regulates ubiquitination, nuclear translocation and activation of IRF7, and thus inhibits a major driver behind type I interferon expression. In searching for the mechanism by which Pellino3 could exert such effects on IRF7 activation we looked beyond its immediate upstream kinases, TBK1 and IKKi, given that loss of Pellino3 did not affect poly(I:C)-induced phosphorylation of TBK1. A previous study has shown that Epstein-Barr virus LMP1 uses TRAF6 to ubiquitinate IRF7 on its last three lysine residues, with such ubiquitination being a prerequisite for IKKi-mediated phosphorylation and activation of IRF7 (ref. 41). TRAF6 also has been shown to fulfill a critically important role in the ubiquitination and activation of IRF7 by TLR7 and TLR9 in pDCs ${ }^{40}$. TRAF6 has a role in the activation of the IFN- $\beta$ promoter by poly(I:C) and EMCV, with the replication of EMCV being considerably enhanced in the absence of TRAF6 (ref. 42). These reports closely mirrored our findings in Pellino3deficient mice and promote TRAF6 as the lead target for Pellino3.

We found that TLR3 signaling promoted the interaction of Pellino3 with TRAF6, leading to strong ubiquitination of TRAF6, which depended on the functional RING-like domain in Pellino3. These observations strongly suggest that Pellino3 acts as the immediate E3 ubiquitin ligase for TRAF6. However, this does not exclude the possibility that Pellino3 associates with TRAF6 and stimulates the autoubiquitination of TRAF6. In an in vitro ubiquitination assay, we did not observe direct ubiquitination of TRAF6 by recombinant Pellino3. This may be due to recombinant Pellino3 lacking some prerequisite modification or ancillary protein to manifest its E3 ligase activity to TRAF6. Indeed the E3 ligase catalytic activity of Pellino proteins is strongly enhanced by phosphorylation ${ }^{24-28}$, and in the context of TLR3 signaling it is especially interesting to note that Pellino1 is induced and activated by TBK1 and $\mathrm{IKKi}^{26}$. We showed here that the expression of Pellino3 similarly depends on TBK1-IKKi. Thus it is plausible to extrapolate a model where TLR3 signaling uses TBK1 and IKKi, in an analogous manner to Pellino1, to phosphorylate and activate Pellino3, thus leading to ubiquitination of TRAF6.

We also propose that Pellino3-mediated ubiquitination of TRAF6 negatively influences the ability of TRAF6 to ubiquitinate IRF7. Although the ubiquitination of TRAF6 has been originally proposed to serve to bring TAK1 and IKK complexes into close proximity, thus allowing for IKK activation and downstream activation of NF- $\mathrm{KB}^{43}$, this model has been questioned recently. Thus, a mutant form of TRAF6 that is refractory to ubiquitination can still trigger activation of $\mathrm{NF}-\kappa \mathrm{B}^{44}$, and TRAF6 can generate unanchored polyubiquitin chains to trigger downstream activation of $\mathrm{NF}-\mathrm{\kappa B}^{45}$. We now propose a new role for ubiquitination of TRAF6 in that it suppresses the ubiquitination of IRF7 by decreasing the interaction of TRAF6 with IRF7. It is intriguing that Pellino3 should counterregulate interferon expression by targeting the TRAF6-IRF7 interaction, as the Thogoto virus uses its ML protein as a virulence factor to inhibit the expression of type I interferons by also blocking the interaction of TRAF6 with IRF7 (ref. 46). This highlights a critical involvement for TRAF6-mediated ubiquitination of IRF7 in the induction of type I interferons. Given such an important role, it is not surprising that a physiological regulatory system modulates this part of the pathway to control type I interferon expression, whereas viruses target this pathway to subvert antiviral innate immunity.

Pellino proteins are very highly conserved throughout evolution, and so the notion of a Pellino gene being evolutionarily retained to counter a key antiviral strategy of producing type I interferon appears counterintuitive. However, the expression of type I interferons must be tightly controlled because excessive production can contribute to autoimmune inflammatory diseases ${ }^{47}$. It will be interesting to explore whether naturally occurring defective forms or expression of Pellino3 may provide a genetic basis to some cases of interferon-driven inflammatory disease. Such studies in conjunction with the present findings will help understand the physiological and pathological roles of the Pellino family.

\section{METHODS}

Methods and any associated references are available in the online version of the paper. 
Note: Supplementary information is available in the online version of the paper.

\section{ACKNOWLEDGMENTS}

This work was funded by Science Foundation Ireland (07/IN.1/B972) and the Health Research Board of Ireland (under grant PhD/2007/09). We thank B. Cloak and S.Worrell for technical assistance with photomicroscopy, and M. Healy for assistance with flow cytometry.

\section{AUTHOR CONTRIBUTIONS}

J.S., R.J. and M.M. developed the concept, designed and performed experiments, analyzed data and prepared the figures; N.D., S.Y., B.W. and L.S.T. designed and performed experiments and analyzed data; J.J.C. performed general pathology screening and the pathology-related experiments on heart samples; B.P.M. designed and supervised the EMCV infection studies; P.N.M. conceived the study, supervised the project, analyzed data and wrote the manuscript.

\section{COMPETING FINANCIAL INTERESTS}

The authors declare no competing financial interests.

Published online at http://www.nature.com/doifinder/10.1038/ni.2429.

Reprints and permissions information is available online at http://www.nature.com/ reprints/index.html.

1. Moynagh, P.N. TLR signalling and activation of IRFs: revisiting old friends from the NF-кB pathway. Trends Immunol. 26, 469-476 (2005).

2. Medzhitov, R. et al. MyD88 is an adaptor protein in the hToll/IL-1 receptor family signaling pathways. Mol. Cell 2, 253-258 (1998).

3. Li, S., Strelow, A., Fontana, E.J. \& Wesche, H. IRAK-4: a novel member of the IRAK family with the properties of an IRAK-kinase. Proc. Natl. Acad. Sci. USA 99 5567-5572 (2002).

4. Cao, Z., Xiong, J., Takeuchi, M., Kurama, T. \& Goeddel, D.V. TRAF6 is a signal transducer for interleukin-1. Nature 383, 443-446 (1996).

5. Suzuki, N. et al. Severe impairment of interleukin-1 and Toll-like receptor signalling in mice lacking IRAK-4. Nature 416, 750-756 (2002).

6. Wang, C. et al. TAK1 is a ubiquitin-dependent kinase of MKK and IKK. Nature 412, 346-351 (2001).

7. Moynagh, P.N. The NF-kB pathway. J. Cell Sci. 118, 4589-4592 (2005).

8. Yamamoto, M. et al. Cutting edge: a novel Toll//L-1 receptor domain-containing adapter that preferentially activates the IFN- $\beta$ promoter in the Toll-like receptor signaling. J. Immunol. 169, 6668-6672 (2002).

9. Cusson-Hermance, N., Khurana, S., Lee, T.H., Fitzgerald, K.A. \& Kelliher, M.A Rip1 mediates the Trif-dependent toll-like receptor 3- and 4-induced NF- $\mathrm{KB}$ activation but does not contribute to interferon regulatory factor 3 activation. J. Biol. Chem. 280, 36560-36566 (2005).

10. Meylan, E. et al. RIP1 is an essential mediator of Toll-like receptor 3-induced NF-KB activation. Nat. Immunol. 5, 503-507 (2004).

11. Sasai, M. et al. Direct binding of TRAF2 and TRAF6 to TICAM-1/TRIF adaptor participates in activation of the Toll-like receptor 3/4 pathway. Mol. Immunol. 47 1283-1291 (2010).

12. Sato, S. et al. Toll/IL-1 receptor domain-containing adaptor inducing IFN- $\beta$ (TRIF) associates with TNF receptor-associated factor 6 and TANK-binding kinase 1 , and activates two distinct transcription factors, NF-KB and IFN-regulatory factor-3, in the Toll-like receptor signaling. J. Immunol. 171, 4304-4310 (2003).

13. Jiang, Z., Mak, T.W., Sen, G. \& Li, X. Toll-like receptor 3-mediated activation of NF-KB and IRF3 diverges at Toll-IL-1 receptor domain-containing adapter inducing IFN- $\beta$. Proc. Natl. Acad. Sci. USA 101, 3533-3538 (2004).

14. Gohda, J., Matsumura, T. \& Inoue, J. Cutting edge: TNFR-associated factor (TRAF) 6 is essential for MyD88-dependent pathway but not toll/IL-1 receptor domaincontaining adaptor-inducing IFN- $\beta$ (TRIF)-dependent pathway in TLR signaling. J. Immunol. 173, 2913-2917 (2004).

15. Hacker, H. et al. Specificity in Toll-like receptor signalling through distinct effector functions of TRAF3 and TRAF6. Nature 439, 204-207 (2006).

16. Fitzgerald, K.A. et al. IKKE and TBK 1 are essential components of the IRF3 signaling pathway. Nat. Immunol. 4, 491-496 (2003).

17. Stetson, D.B. \& Medzhitov, R. Type I interferons in host defense. Immunity 25 373-381 (2006).

18. Theofilopoulos, A.N., Baccala, R., Beutler, B. \& Kono, D.H. Type I interferons $(\alpha / \beta)$ in immunity and autoimmunity. Annu. Rev. Immunol. 23, 307-336 (2005).

19. Moynagh, P.N. The Pellino family: IRAK E3 ligases with emerging roles in innate immune signalling. Trends Immunol. 30, 33-42 (2009).
20. Schauvliege, R., Janssens, S. \& Beyaert, R. Pellino proteins: novel players in TLR and IL-1R signalling. J. Cell. Mol. Med. 11, 453-461 (2007).

21. Lin, C.C., Huoh, Y.S., Schmitz, K.R., Jensen, L.E. \& Ferguson, K.M. Pellino proteins contain a cryptic FHA domain that mediates interaction with phosphorylated IRAK1. Structure 16, 1806-1816 (2008).

22. Schauvliege, R., Janssens, S. \& Beyaert, R. Pellino proteins are more than scaffold proteins in TLR/IL-1R signalling: a role as novel RING E3-ubiquitin-ligases. FEBS Lett. 580, 4697-4702 (2006).

23. Butler, M.P., Hanly, J.A. \& Moynagh, P.N. Kinase-active interleukin-1 receptorassociated kinases promote polyubiquitination and degradation of the Pellino family: direct evidence for PELLINO proteins being ubiquitin-protein isopeptide ligases. J. Biol. Chem. 282, 29729-29737 (2007).

24. Ordureau, A. et al. The IRAK-catalysed activation of the E3 ligase function of Pellino isoforms induces the Lys63-linked polyubiquitination of IRAK1. Biochem. J. 409, 43-52 (2008).

25. Smith, $\mathrm{H}$. et al. Identification of the phosphorylation sites on the E3 ubiquitin ligase Pellino that are critical for activation by IRAK1 and IRAK4. Proc. NatI. Acad. Sci. USA 106, 4584-4590 (2009).

26. Smith, H. et al. The role of TBK1 and IKKepsilon in the expression and activation of Pellino 1. Biochem. J. 434, 537-548 (2011).

27. Goh, E.T. et al. Identification of the protein kinases that activate the E3 ubiquitin ligase Pellino 1 in the innate immune system. Biochem. J. 441, 339-346 (2012).

28. Strelow, A., Kollewe, C. \& Wesche, H. Characterization of Pellino2, a substrate of IRAK1 and IRAK4. FEBS Lett. 547, 157-161 (2003).

29. Kim, J.H. et al. Pellino-1, an adaptor protein of interleukin-1 receptor/toll-like receptor signaling, is sumoylated by Ubc9. Mol. Cells 31, 85-89 (2011).

30. Jiang, Z. et al. Pellino 1 is required for interleukin-1 (IL-1)-mediated signaling through its interaction with the IL-1 receptor-associated kinase 4 (IRAK4)-IRAKtumor necrosis factor receptor-associated factor 6 (TRAF6) complex. J. Biol. Chem. 278, 10952-10956 (2003).

31. Yu, K.Y. et al. Cutting edge: mouse pellino-2 modulates IL-1 and lipopolysaccharide signaling. J. Immunol. 169, 4075-4078 (2002).

32. Jensen, L.E. \& Whitehead, A.S. Pellino2 activates the mitogen activated protein kinase pathway. FEBS Lett. 545, 199-202 (2003).

33. Jensen, L.E. \& Whitehead, A.S. Pellino3, a novel member of the Pellino protein family, promotes activation of c-Jun and Elk-1 and may act as a scaffolding protein. J. Immunol. 171, 1500-1506 (2003).

34. Butler, M.P., Hanly, J.A. \& Moynagh, P.N. Pellino3 is a novel upstream regulator of p38 MAPK and activates CREB in a p38-dependent manner. J. Biol. Chem. 280 27759-27768 (2005).

35. Chang, M., Jin, W. \& Sun, S.C. Peli1 facilitates TRIF-dependent Toll-like receptor signaling and proinflammatory cytokine production. Nat. Immunol. 10, 1089-1095 (2009).

36. Chang, M. et al. The ubiquitin ligase Peli1 negatively regulates $\mathrm{T}$ cell activation and prevents autoimmunity. Nat. Immunol. 12, 1002-1009 (2011).

37. Moynagh, P.N. Peli1 (rel)ieves autoimmunity. Nat. Immunol. 12, 927-929 (2011).

38. Gitlin, L. et al. Essential role of mda-5 in type I IFN responses to polyriboinosinic: polyribocytidylic acid and encephalomyocarditis picornavirus. Proc. Natl. Acad. Sci. USA 103, 8459-8464 (2006).

39. Hardarson, H.S. et al. Toll-like receptor 3 is an essential component of the innate stress response in virus-induced cardiac injury. Am. J. Physiol. Heart Circ. Physiol. 292, H251-H258 (2007).

40. Kawai, T. et al. Interferon-alpha induction through Toll-like receptors involves a direct interaction of IRF7 with MyD88 and TRAF6. Nat. Immunol. 5, 1061-1068 (2004).

41. Ning, S., Campos, A.D., Darnay, B.G., Bentz, G.L. \& Pagano, J.S. TRAF6 and the three C-terminal lysine sites on IRF7 are required for its ubiquitination-mediated activation by the tumor necrosis factor receptor family member latent membrane protein 1. Mol. Cell. Biol. 28, 6536-6546 (2008).

42. Konno, $\mathrm{H}$. et al. TRAF6 establishes innate immune responses by activating NF- $\mathrm{KB}$ and IRF7 upon sensing cytosolic viral RNA and DNA. PLOS ONE 4, e5674 (2009).

43. Chen, Z.J. Ubiquitin signalling in the NF-KB pathway. Nat. Cell Biol. 7, 758-765 (2005).

44. Walsh, M.C., Kim, G.K., Maurizio, P.L., Molnar, E.E. \& Choi, Y. TRAF6 autoubiquitination-independent activation of the NF-KB and MAPK pathways in response to IL-1 and RANKL. PLOS ONE 3, e4064 (2008).

45. Xia, Z.P. et al. Direct activation of protein kinases by unanchored polyubiquitin chains. Nature 461, 114-119 (2009).

46. Buettner, N. et al. Thogoto virus ML protein is a potent inhibitor of the interferon regulatory factor-7 transcription factor. J. Gen. Virol. 91, 220-227 (2010).

47. Choubey, D. \& Moudgil, K.D. Interferons in autoimmune and inflammatory diseases: regulation and roles. J. Interferon Cytokine Res. 31, 857-865 (2011). 


\section{ONLINE METHODS}

Plasmids and reagents. Mouse Pellino3 was cloned from embryo brain and into the retroviral MSCV2.2-IRES-GFP vector. Human TRAF6 C70A and K124R mutants and Traf6 ${ }^{-1-}$ MEFs were from A. Bowie (Trinity College Dublin). Immortalized wild-type, Ticam $1^{-1-}, \mathrm{Tbk}^{-1-}$ and $I k b k e^{-/-} \mathrm{BMDMs}$ were from K. Fitzgerald (University of Massachusetts Medical School). FLT3 ligand was from C. Jefferies (Royal College of Surgeons Ireland). Anti-TRAF6 (sc-7221), anti-ubiquitin (P4D1), anti-IRF3 (sc-9082) and anti-IRF7 (F-1) antibodies for coimmunoprecipitation were from Santa Cruz Biotechnology. The anti-IRF7 antibody (ab109255) for immunoblotting was from Abcam. Anti-phospho IKB $\alpha$ (9246), anti-phospho IKK (2694), anti-IKK (2684) anti-phospho TBK1 (D52C2), anti-TBK1 (3013), anti-phospho IRF3 (4D4G), anti-phospho IRF7 (5184) and anti-Myc (9B11) antibodies were from Cell Signaling Technology. The anti-phospho-serine antibody (AB1603) was from Millipore. The anti- $\beta$-actin antibody (AC-15) was from Sigma. The customized anti-human Pellino3 antibody was from GenScript. LPS was from Enzo Life Sciences and other TLR ligands were from Invivogen.

Mice. Peli3 ${ }^{-/-}$mice were generated by Taconic Artemis using proprietary technology (Supplementary Fig. 1a). To generate constitutive Peli3 ${ }^{-/-}$mice, mice that were heterozygous for the targeted allele were bred with mice containing Cre recombinase regulated by the Rosa26 locus (C57BL/6 Gt(ROSA)26Sortm 16 (Cre)Arte). This results in the deletion of exon 3 and loss of function of the Peli3 gene by generating a frame shift in all downstream exons. The Cre transgene was removed by breeding the resulting Peli3 ${ }^{+/-}$mice with C57BL/ 6 mice during colony expansion. Mice were genotyped by PCR analysis of DNA isolated from ear punches using primers 'a', CCCAACATAGGTGTTTCCTCTCC; 'b', GTGCATACACATTCATGCAAGC; 'c', GACACGTGTGGAGATAATGAGG and 'd', ACCCAGGCACAAGTCAAGC. All animal experiments were performed in accordance with the regulations and guidelines of the Irish Department of Health and protocols approved by the Research Ethics committee of National University of Ireland Maynooth.

EMCV infection. We injected 50 PFU or 250 PFU of EMCV intraperitoneally into 6-week-old wild-type or Peli3 ${ }^{-1-}$ mice. The day of virus inoculation was defined as day 0 . Mice that became moribund were considered to have reached the end point of the experiment and were killed. Mice were killed $12 \mathrm{~d}$ or $14 \mathrm{~d}$ after infection, and ratio of heart weight to body weight was measured. Hearts were then fixed, sectioned at $5 \mu \mathrm{m}$ and stained with hematoxylin and eosin The pathologist (J.J.C.) was blinded to the genetic and infectious status of the mice. For cytokine and EMCV replicase analysis, mice were inoculated with 50 PFU) EMCV and allowed recover for $4 \mathrm{~d}$. Mice were killed and total RNA was extracted from tissue using TRI reagent (Sigma).

Cell culture. Peli3 ${ }^{+/-}$mice were bred to generate wild-type and Peli3-1embryos and MEFs. Bone marrow cells were maintained in macrophage-colony stimulating factor (M-CSF; $20 \mathrm{ng} / \mathrm{ml}$ ) for 5-7 d, or granulocyte macrophagecolony stimulating factor (GM-CSF; $10 \mathrm{ng} / \mathrm{ml}$ ) or Fms-related tyrosine kinase 3 ligand (FLT3L; $10 \mathrm{ng} / \mathrm{ml}$ ) for 8-10 d to generate BMDMs, mDC or pDC cells, respectively. $\mathrm{pDCs}$ were enriched using a $\mathrm{pDC}$ isolation kit (Miltenyi Biotec).

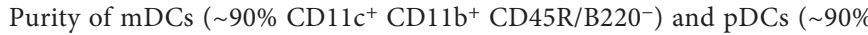

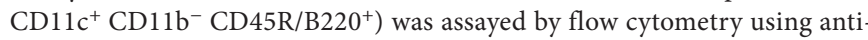
CD11c (N418), anti-CD11b (M1/70) and anti-CD45R/B220 (RA3-6B2) on live cells as determined by 7-AAD Viability Staining Solution (eBioscience).

Generation of peritoneal exudate cells (PECs). Age-matched and weightmatched wild-type and Peli3 ${ }^{-1-}$ mice were injected intraperitoneally with $1 \mathrm{mg} / \mathrm{kg}$ poly(I:C) for $48 \mathrm{~h}$. PECs were isolated and relative expression of mRNAs encoding IFN- $\beta$, RANTES and TNF was determined by quantitative real-time RT-PCR.

ELISA and type I interferon bioassay. Mouse cells were seeded $\left(5 \times 10^{5}\right.$ cells/ml; $200 \mu \mathrm{l}$ ) in 96-well plates and stimulated as indicated. Conditioned medium was measured for levels of TNF, IL-6 (DuoSet kits; R\&D Systems), CXCL10 (ELISA development kit; Peprotech) and IFN- $\alpha$ (mouse IFN $\alpha$ Platinum ELISA; eBioscience) and mouse type I interferon by blue sensor cells (Invitrogen) according to manufacturer's instructions. IFN- $\beta$ protein levels were assayed by an in-house sandwich ELISA system. Conditioned supernatants from U373 shRNA stable cell lines were assayed for RANTES, CXCL10 and IL-6 (DuoSet kits; R\&D Systems).

Elecrophoretic mobility shift assay. MEFs were grown in 6-well plates for $24 \mathrm{~h}$. Cells were then stimulated with $25 \mu \mathrm{g} / \mathrm{ml}$ poly(I:C). Nuclear extracts were generated as previously described ${ }^{48}$ and incubated with an oligonucleotide containing the IFN- $\beta$ PRD I/III-binding site $5^{\prime}$-GTAAATGACATAGGAAAA CTGAAAGGGAGAAGTGAAAGTGG-3' and labeled with IRDye 700 according to the manufacturer's instructions.

Immunoprecipitation studies. Primary MEFs or mDCs were seeded in cell-culture $90-\mathrm{mm}$ dishes $\left(5 \times 10^{5}\right.$ cells $/ \mathrm{ml}$ or $1 \times 10^{6}$ cells $/ \mathrm{ml}$, respectively) and stimulated with poly(I:C) for designated times. Cells were washed in $1 \mathrm{ml}$ of ice-cold PBS and lysed in $200 \mu \mathrm{l}$ of radioimmunoprecipitation (RIPA) buffer: $50 \mathrm{mM}$ Tris, $150 \mathrm{mM} \mathrm{NaCl}, 0.1 \% \mathrm{SDS}(\mathrm{w} / \mathrm{v}), 0.5 \%$ sodium deoxycholate (w/v), $1 \%$ Triton X-100 (v/v) containing $1 \mathrm{mM} \mathrm{Na}_{3} \mathrm{VO}_{4}, 1 \mathrm{mM}$ dithiothreitol, $1 \mathrm{mM}$ phenylmethylsulfonyl fluoride and complete protease inhibitor mixture (Roche). Cell lysates were treated with $1 \%$ SDS and incubated at $95{ }^{\circ} \mathrm{C}$ for $5 \mathrm{~min}$ to dissociate protein-protein interactions. Samples were then diluted tenfold with lysis buffer before immunoprecipitation and immunoblotting using indicated antibodies.

Coimmunoprecipitation studies. MEFs or U373 cells were seeded in 90-mm dishes $\left(5 \times 10^{5}\right.$ cells $\left./ \mathrm{ml}\right)$ and stimulated with poly(I:C) for indicated durations. Cells were washed in $1 \mathrm{ml}$ ice-cold PBS and lysed in $250 \mu \mathrm{l}$ of lysis buffer (50 mM Hepes pH 7.5, 1 mM EDTA, 10\% glycerol (v/v), 0.05\% CHAPS (w/v), $0.5 \%$ Triton X-100 (v/v), $250 \mathrm{mM} \mathrm{NaCl}$ containing $1 \mathrm{mM} \mathrm{Na}_{3} \mathrm{VO}_{4}, 1 \mathrm{mM}$ dithiothreitol, $1 \mathrm{mM}$ phenylmethylsulfonyl fluoride and complete protease inhibitor mixture). Lysates were processed as described above for immunoprecipitation studies.

Transfection and luciferase reporter assays. In all transfection-based studies (including knockdown experiments with $20 \mathrm{nM}$ siRNA and $2 \mu \mathrm{g} / \mathrm{ml}$ shRNA) in HEK293 cells we used Lipofectamine 2000 (Invitrogen) according to the manufacturer's instructions. For luciferase reporter assays, HEK293 TLR3 cells were seeded $\left(1.5 \times 10^{5}\right.$ cells $\left./ \mathrm{ml} ; 200 \mu \mathrm{l}\right)$ in 96-well plates and transfected with constructs encoding IFN- $\beta$, IFN- $\alpha 4$ or PRDI-III-regulated firefly luciferase $(80 \mathrm{ng})$ or pFR firefly luciferase reporter construct (60 ng) with Gal4IRF3 (30 ng) or Gal4-IRF7 (25 ng), TK Renilla luciferase reporter construct (phRL-TK; 20 ng; Promega Biosciences) and Pellino3 expression constructs (1-20 ng). Total DNA was kept constant (200 ng/well) using the appropriate empty vector. Cells were treated as indicated and cell lysates assayed for firefly luciferase activity and normalized for transfection efficiency using TK Renilla luciferase activity.

For studies using transfection-mediated delivery of poly(I:C), BMDMs were seeded $\left(5 \times 10^{5}\right.$ cells $\left./ \mathrm{ml} ; 200 \mu \mathrm{l}\right)$ in 96 -well plates and grown for $24 \mathrm{~h}$. Cells were then transfected using Lipofectamine 2000 with $5 \mu \mathrm{g} / \mathrm{ml}$ poly(I:C) and incubated for $24 \mathrm{~h}$. Conditioned supernatant was assayed by ELISA.

Lentiviral production and transduction. PELI3-specific shRNA was used to suppress endogenous expression of Pellino3 in U373 cells as previously described $^{49}$. Knockdown efficiency was assessed by RT-PCR.

Retroviral production and transduction. MEFs were seeded $\left(3 \times 10^{5}\right.$ cells $/ \mathrm{ml}$; $3 \mathrm{ml}$ ) into 6-well plates (for quantitative PCR) or in $90 \mathrm{~mm}$ dishes (for immunoprecipitation) and were transduced with a MSCV retroviral plasmid encoding mouse (m)Pellino3 or the mPellino3 RING mutant as described ${ }^{49}$.

Quantitative PCR. RNA was extracted from cells using Tri-Reagent (Sigma) and cDNA generated using AMV reverse transcriptase (Promega). Samples were assayed by quantitative real time PCR using the CFB322001G Opticon thermal cycler (Bio-Rad Laboratories) with Brilliant SYBR Green QPCR master mix (Stratagene) and the following primers: mIFN- $\alpha$ forward, GGCTTGACACTCCTGGTACAAATGAG; mIFN- $\alpha$ reverse, CAGCACATTGGCAGAGGAAGACAG; hIFN- $\alpha$ forward, GAA ATACTTCCAAAGAATCACTCT; hIFN- $\alpha$ reverse, GATCTCATGATTT 
CTGCTCTGACA; mIFN- $\beta$ forward, GGAGATGACGGAGAAGATGC; mIFN- $\beta$ reverse, CCCAGTGCTGGAGAAATTGT; hIFN- $\beta$, forward, AACT GCAACCTTTCGAAGCC; hIFN- $\beta$ reverse, TGTCGCCTACTACCTGTTG TGC; mTNF- $\alpha$ forward, CATCTTCTCAAAATTCGAGTGACAA; mTNF- $\alpha$ reverse, TGGGAGTAGACAAGGTACAACCC; hIL6 forward, AGCCACTCA CCTCTTCAGAACGAA; hIL6 reverse, CAGTGCCTCTTTGCTGCTTTCA CA; mPellino3 forward, ACATGCCAACGGAGTGAAGC; mPellino3 reverse, AGCGGCCAATCTGGAACAT; mRANTES forward, GGAGATGAGCTA GGATAGAGGG; mRANTES reverse, TGCCCATTTTCCCAGGACCG; hRANTES forward, TGCCTGTTTCTGCTTGCTCTTGTC; hRANTES reverse, TGTGGTAGAATCTGGGCCCTTCAA; mCXCL10 forward, GACGG TCCGCTGCAACTG; mCXCL10 reverse, GCTTCCCTATGGCCCTCATT; hCXCL10 forward, ATTATTCCTGCAAGCCAATTTTG; hCXCL10 reverse, TCACCCTTCTTTTTCATTGTAGCA; mHPRT forward, GCTTGCTGGTG AAAAGGACCTCTCGAAG; mHPRT reverse, CCCTGAAGTACTCATTAT AGTCAAGGGCAT; hHPRT forward, AGCTTGCTGGTGAAAAGGAC; and hHPRT reverse, TTATAGTCAAGGGCATATCC.

Confocal microscopy. MEFs were seeded $\left(3 \times 10^{5}\right.$ cells $\left./ \mathrm{ml}\right)$ on polyL-lysine-coated slides and treated with $25 \mu \mathrm{g} / \mathrm{ml}$ poly(I:C) for $90 \mathrm{~min}$. Cells were washed three times in chilled PBS $(500 \mu \mathrm{l})$, fixed with $4 \%(\mathrm{v} / \mathrm{v})$ paraformaldehyde $(500 \mu \mathrm{l}$ ) for $20 \mathrm{~min}$, permeabilized with $0.2 \%$ Triton X-100 for 10 min and blocked with $10 \%$ goat serum (Vector Laboratories) for $2 \mathrm{~h}$. Cells were incubated overnight at $4{ }^{\circ} \mathrm{C}$ with anti-IRF7 antibody (1:200; F-1, Santa Cruz), washed and incubated with goat anti-mouse Alexa Fluor 488-labeled secondary antibody (1:500; A-11001, Invitrogen). Nuclei were stained with DAPI ( $1.5 \mu \mathrm{g} / \mathrm{ml}$; Sigma) for $2 \mathrm{~min}$. Cells were mounted and confocal images were captured using the $\times 63$ objective lens ( $\times 630$ total magnification) on the UV Zeiss 510 Meta System laser scanning microscope and analyzed using the LSM 5 browser imaging software.
Isolation of nuclear fractions. BMDMs were seeded $\left(5 \times 10^{5}\right.$ cells $\left./ \mathrm{ml}\right)$ and treated with $5 \mu \mathrm{g} / \mathrm{ml}$ poly(I:C) durations indicated for each experiment. Cells were resuspended for $10 \mathrm{~min}$ on ice in buffer A: $10 \mathrm{mM}$ HEPES pH 7.9, $10 \mathrm{mM}$ $\mathrm{KCl}, 0.1 \mathrm{mM}$ EDTA, $0.1 \mathrm{mM}$ EGTA, $0.1 \mathrm{mM} \mathrm{Na} 3 \mathrm{VO}_{4}$ followed by additional washes in buffer $\mathrm{A}$. The nuclear pellet was resuspended in three volumes of ice-cold buffer B: $20 \mathrm{mM}$ HEPES pH 7.9, $10 \mathrm{mM} \mathrm{KCl,} 1 \mathrm{mM}$ EDTA, $1 \mathrm{mM}$ EGTA, $420 \mathrm{mM} \mathrm{NaCl}$ and $20 \%$ glycerol. Samples were centrifuged at $12,000 \mathrm{~g}$ for $10 \mathrm{~min}$, and the supernatants constituted nuclear fractions that were immunoblotted using anti-IRF7 (ab109255, Abcam), anti-IRF3 (sc-9082, Santa Cruz) and anti-nucleolin (sc-13057, Santa Cruz) antibodies.

Chromatin immunoprecipitation assay. Chromatin immunoprecipitation (ChIP) assays were performed essentially as previously described ${ }^{48}$ using anti-IRF7 antibody (ab109255, Abcam) or mouse IgG (12-371, Upstate Laboratories). Standard PCR was conducted with specific primers (5'-AAGACAAAATGACAGAGGA- $3^{\prime}$ and reverse, $5^{\prime}$-ATGGTCCTT TCTGCCTCA- $3^{\prime}$ ) designed to amplify the enhanceosome region of the Ifnb1 promoter.

Statistical analysis. Data are the means \pm s.e.m. of triplicate determinations from three independent experiments. For comparison between two groups, Student's two-tailed paired $t$-test was used. For comparison between two or more groups one-way ANOVA was applied. $P<0.05$ was considered significant.

48. Curran, N.M. et al. The synthetic cannabinoid R(+)WIN 55,212-2 inhibits the interleukin-1 signaling pathway in human astrocytes in a cannabinoid receptorindependent manner. J. Biol. Chem. 280, 35797-35806 (2005).

49. Mellett, M., Atzei, P., Jackson, R., O'Neill, L.A. \& Moynagh, P.N. Mal mediates TLRinduced activation of CREB and expression of IL-10. J. Immunol. 186, 4925-4935 (2011). 